Activity Index-2K(SLEDAI-2K) was recorded. Matched saliva, serum, and urine were collected, and the levels of colonystimulating factor (CSF)-1, tumor necrosis factor (TNF)- $\alpha$, interferon- $\gamma$-induced protein (IP)-10, monocyte chemoattractant protein (MCP)-1 and calprotectin were analysed by enzymelinked immunosorbent assays (ELISA) and Bead-based Multiplex immunoassay.

Results TNF- $\alpha$, IP-10 and MCP-1 in saliva, serum and urine, and CSF-1 in saliva and serum provided diagnostic ability to distinguish SLE from controls (area under the curve $>0.659$, $\mathrm{p}<0.05$ for all). CSF-1, TNF- $\alpha$, IP-10 and MCP-1 in saliva, serum and urine as well as calprotectin in saliva and urine were increased in SLE patients. Levels of CSF-1 in saliva, serum and urine, IP-10 in serum and urine, and calprotectin in saliva and urine, as well as TNF- $\alpha$, IP-10 and MCP-1 in urine correlated positively with measures of disease activity. TNF- $\alpha$, IP-10 and MCP-1 in urine and CSF-1 and IP-10 in serum were elevated in patients with active renal disease ( $\mathrm{p}<0.05$ all comparisons).

Conclusion The proposed biomarkers provide diagnostic ability to distinguish SLE from controls and they also reflect disease activity. This is a promising result suggesting that in some situations either saliva or urine samples might be capable to be an alternative to serum when diagnosing and monitoring patients with SLE.

\section{P38 DESCRIPTIVE ANALYSIS OF PATIENTS POSITIVE FOR ANTI-PCNA ANTIBODIES}

${ }^{1}$ Beatriz Samões, ${ }^{2}$ Maria Figueiredo, 'Diogo Fonseca, ${ }^{1}$ Miguel Guerra, ${ }^{1}$ Romana Vieira, ${ }^{1}$ Taciana Videira, 'Joana Abelha-Aleixo, 'Patrícia Pinto, ${ }^{2}$ Ana Paula Cruz. 'Rheumatology Dept., Centro Hospitalar de Vila Nova de Gaia e Espinho (CHVNG), Vila Nova de Gaia; ${ }^{2}$ Clinical Pathology Dept., CHVNG, Vila Nova de Gaia, Portugal

\subsection{6/lupus-2020-eurolupus.86}

Background Proliferating cell nuclear antigen (PCNA) antibodies are a rare type of antinuclear antibodies (ANA), historically considered highly specific for systemic lupus erythematosus (SLE). Recent studies showed they can be present in other autoimmune diseases, viral infections or tumors. Our aim was to clinically describe patients positive for anti-PCNA antibodies.

Methods We retrospectively identified patients with a PCNA pattern by indirect immunofluorescence (IIF), subsequently confirmed by immunoblot, selected among patients tested for ANA, between January 2006 and August 2019. Clinical and analytical data were obtained from medical records.

Results 11014 samples (excluding follow-up tests) were tested for ANA, 2642 (23.99\%) were positive (titer $>1$ / $160), 26(0.98 \%)$ had a PCNA pattern by IIF but only 6 $(0.23 \%)$ were anti-PCNA positive. Mean patients' age was $51.5 \pm 17.93$ years-old and the majority were female $(83 \%)$. Half had SLE (including one Overlap Syndrome). Other autoimmune diseases included Antiphospholipid syndrome $[n=2,33 \%]$, Systemic Sclerosis $[n=1,17 \%]$ and Behçet Disease $[n=1,17 \%]$. One patient developed metastasis from an occult neoplasia and none had viral disease. Almost all patients had cutaneous manifestations [ $n=5,83 \%]$. Half had articular $[n=3,50 \%]$ and neurological manifestations $[n=3$, 50\%]. Median ESR and RCP were slightly elevated. None had complement consumption. Mean GFR was normal, with one patient showing low level of proteinuria. Anti-dsDNA, anti-SSA, anti-M2 and anti-cardiolipin were also found in the sera. Two patients were under hydroxychloroquine $[\mathrm{n}=2,33 \%]$ and one under prednisolone $10 \mathrm{mg}[\mathrm{n}=1$, 17\%] (table 1).

\begin{tabular}{ll} 
Abstract P38 Table 1 & \multicolumn{1}{c}{ Descriptive analysis of the clinical and } \\
analytical data of the 6 patients positive for anti-PCNA \\
\hline Disease duration (years), median [min, max] & $1[1,17]$ \\
Autoimmune disorders: & \\
Systemic lupus erythematous (SLE), $\mathrm{n}(\%)$ & $2(33)$ \\
Secondary Antiphospholipid syndrome, $\mathrm{n}(\%)$ & $2(33)$ \\
Systemic Sclerosis (SyS), $\mathrm{n}(\%)$ & $1(17)$ \\
Overlap syndrome (SLE + SyS), $\mathrm{n}(\%)$ & $1(17)$ \\
Behçet disease, $\mathrm{n}(\%)$ & $1(17)$ \\
No evidence of autoimmune disorder, $\mathrm{n}(\%)$ & $1(17)$ \\
Other diseases: & \\
Malignancies, $\mathrm{n}(\%)$ & $1(17)$ \\
Viral infections, $\mathrm{n}(\%)$ & $0(0)$ \\
Clinical manifestations: & \\
Skin, $\mathrm{n}(\%)$ & $5(83)$ \\
Joints, $\mathrm{n}(\%)$ & $3(50)$ \\
Neurological, $\mathrm{n}(\%)$ & $3(50)$ \\
Constitutional, $\mathrm{n}(\%)$ & $2(33)$ \\
Renal, $\mathrm{n}(\%)$ & $2(33)$ \\
Muscle, $\mathrm{n}(\%)$ & $1(17)$ \\
Sicca, $\mathrm{n}(\%)$ & $1(17)$ \\
Analytical data (at time of PCNA positive test) & \\
RCP (mg/dL), median [min, max] & $0.86[0.04,22.89]$ \\
ESR (mm/h), median [min, max] & $38.5[6,113]$ \\
Number of patients with complement consumption (\%) & $0(0)$ \\
GFR (MDRD Equation, mL/min/1.73 m²), mean \pm SD & $92.9 \pm 44.8$ \\
Number of patients with proteinuria, $\mathrm{n}(\%)$ & $1(17)$ \\
Serological data & $1(17)$ \\
Positive anti-dsDNA antibodies, $\mathrm{n}(\%)$ & $1(17)$ \\
Positive anti-SSA antibodies, $\mathrm{n}(\%)$ & $1(17)$ \\
Positive anti-Mitochondrial M2 antibodies, $\mathrm{n}(\%)$ & $1(17)$ \\
Positive anti-Cardiolipin antibodies, $\mathrm{n}(\%)$ & \\
\hline &
\end{tabular}

Conclusions Our study confirmed that anti-PCNA antibodies can be present in other autoimmune diseases and are not SLE specific. Given its rare prevalence, evidence about the clinical relevance of anti-PCNA antibodies is lacking. Larger studies are needed to further evaluate its role.

Skin: Malar rash and/or photosensitivity and/or oral/genital ulcers and/or Raynaud phenomenon and/or telangiectasias and/ or microstomy and/or digital ulcers and/or skin sclerosis;

Joints: Arthralgias and/or arthritis;

Neurological: Focal neurological symptoms and/or dementia and/or cerebrovascular disease;

Constitutional: Fatigue and/or weight loss; Renal: serum creatinine elevation and/or proteinuria and/or hematuria; Muscle: Myalgias and/or myositis;

Sicca: Dry mouth/eyes;

RCP: Reactive C protein;

ESR: Erythrocyte sedimentation rate;

GFR: Glomerular filtration rate. 